\title{
New fixed point free nonexpansive maps on weakly compact, convex subsets of $L^{1}[0,1]$
}

\author{
by \\ P. N. Dowling (Oxford, OH), C. J. Lennard (Pittsburgh, PA) and \\ B. TuretT (Rochester, MI)
}

\begin{abstract}
We show that every subset of $L^{1}[0,1]$ that contains the nontrivial intersection of an order interval and finitely many hyperplanes fails to have the fixed point property for nonexpansive mappings.
\end{abstract}

1. Introduction and preliminaries. In 1981, D. Alspach [1] gave the first example of a weakly compact, convex subset of a Banach space that fails the fixed point property for nonexpansive mappings. A modification of Alspach's example by R. Sine [11] was used in [5] to show that every closed, bounded, convex subset of $L^{1}[0,1]$ that contains a nontrivial order interval fails the fixed point property for nonexpansive mappings. In this paper, Alspach's example is used to show that every subset of $L^{1}[0,1]$ that contains the nontrivial intersection of an order interval and finitely many hyperplanes fails to have the fixed point property for nonexpansive mappings. This generalizes the result in [5] and, unlike the previous theorem, also includes Alspach's example.

As usual, $\mathbb{N}$ denotes the set of all positive integers, $\mathbb{Z}$ is the set of all integers and $\mathbb{R}$ denotes the set of all real numbers. A set $K$ has the fixed point property for nonexpansive mappings if every nonexpansive map of $K$ into itself has a fixed point. We refer the reader to the texts of Diestel [3] and Goebel and Kirk [6] for any unexplained terminology.

2. A new fixed point free mapping theorem in $L^{1}[0,1]$. Recall Alspach's construction. Let $C:=\left\{f \in L^{1}[0,1]: 0 \leq f(t) \leq 1\right.$ for all $t \in[0,1]\}$. Now define $T: C \rightarrow C$ by setting, for all $f \in C$,

2000 Mathematics Subject Classification: Primary 47H10, 47H09, 46E30.

Key words and phrases: nonexpansive mapping, fixed point property, convex set, Alspach's map. 


$$
(T f)(t):= \begin{cases}2 f(2 t) \wedge 1 & \text { if } 0 \leq t<1 / 2 \\ (2 f(2 t-1)-1) \vee 0 & \text { if } 1 / 2 \leq t<1 .\end{cases}
$$

Alspach showed that the mapping $T$ is an isometry on $C$ which has only two fixed points: 0 and $\chi_{[0,1]}$. Alspach also showed that $T$ maps the closed convex subset $C_{0}:=\left\{f \in C: \int_{[0,1]} f d m=1 / 2\right\}$ of $C$ into itself. This proves that $T$ is a fixed point free isometry on the weakly compact, convex set $C_{0}$. Here, $m$ denotes Lebesgue measure on the $\sigma$-algebra $\mathcal{M}$ of Lebesgue measurable subsets of $[0,1]$.

Sine [11] considered a modification of Alspach's example by defining $S: C \rightarrow C$ by $S(f):=\chi_{[0,1]}-T f$ for all $f \in C$. Note that $S$ is nonexpansive on $C$, and that the two fixed points of $T$ in $C$, namely 0 and $\chi_{[0,1]}$, are not fixed points of $S$. In fact, Sine proved that $S$ has no fixed points in the set $C$.

For our purpose, it will be useful to construct a somewhat different fixed point free nonexpansive mapping on $C$. To this end, we define a mapping $\Delta$ on $C$ by setting, for all $f \in C$,

$$
(\Delta f)(t):= \begin{cases}f(2 t) & \text { if } 0 \leq t<1 / 2 \\ 1-f(2 t-1) & \text { if } 1 / 2 \leq t<1\end{cases}
$$

Two key properties of $\Delta$ that are easy to check are that $\Delta$ maps $C$ into $C_{0}$ and $\Delta$ is an $L^{1}[0,1]$-isometry on $C$.

Lemma 1. The function $T \Delta: C \rightarrow C_{0} \subseteq C$ is fixed point free on $C$.

Proof. A straightforward calculation shows that for all $f \in C$,

$$
(T \Delta f)(t):= \begin{cases}2 f(4 t) \wedge 1 & \text { if } 0 \leq t<1 / 4 \\ 2(1-f(4 t-1)) \wedge 1 & \text { if } 1 / 4 \leq t<1 / 2, \\ (2 f(4 t-2)-1) \vee 0 & \text { if } 1 / 2 \leq t<3 / 4 \\ (1-2 f(4 t-3)) \vee 0 & \text { if } 3 / 4 \leq t<1\end{cases}
$$

Now, assume, to get a contradiction, that there exists $f \in C$ such that $T \Delta(f)=f$. Then clearly, $f=T \Delta(f) \in C_{0}$. Define sets

$$
\begin{gathered}
A:=[f=0]=\{t \in[0,1]: f(t)=0\}, \quad B:=[f=1], \\
D:=[0<f<1 / 2], \quad E:=[1 / 2<f<1], \quad F:=[f=1 / 2] .
\end{gathered}
$$

Then, with $\dot{U}$ denoting disjoint union and all set equalities modulo sets of measure zero, it follows that

$$
\begin{aligned}
A= & \frac{1}{4} A \dot{\cup}\left(\frac{1}{4}+\frac{1}{4} B\right) \dot{\cup}\left(\frac{1}{2}+\frac{1}{4} A\right) \dot{\cup}\left(\frac{1}{2}+\frac{1}{4} D\right) \\
& \dot{U}\left(\frac{1}{2}+\frac{1}{4} F\right) \dot{\cup}\left(\frac{3}{4}+\frac{1}{4} B\right) \dot{\cup}\left(\frac{3}{4}+\frac{1}{4} E\right) \dot{\cup}\left(\frac{3}{4}+\frac{1}{4} F\right)
\end{aligned}
$$


and

$$
\begin{aligned}
B= & \frac{1}{4} B \dot{\cup} \frac{1}{4} E \dot{\cup} \frac{1}{4} F \dot{\cup}\left(\frac{1}{4}+\frac{1}{4} A\right) \dot{\cup}\left(\frac{1}{4}+\frac{1}{4} D\right) \\
& \dot{U}\left(\frac{1}{4}+\frac{1}{4} F\right) \dot{\cup}\left(\frac{1}{2}+\frac{1}{4} B\right) \dot{U}\left(\frac{3}{4}+\frac{1}{4} A\right) .
\end{aligned}
$$

From these two equalities, it follows that

$$
2(m(A)-m(B))=m(D)+m(E)+2 m(F)=2(m(B)-m(A)) .
$$

Thus, $m(A)=m(B)$ and $m(D)=m(E)=m(F)=0$ and

$$
f=\chi_{B} \text { a.e. and } m(B)=1 / 2 .
$$

Moreover, ignoring sets of measure zero and substituting the above equations for $A$ and $B$ into the right-hand side of the equation for $B$ shows, upon iteration, that for all $\nu \in \mathbb{N}$ and all $j \in\left\{0, \ldots, 4^{\nu}-1\right\}$,

$$
B \cap\left[\frac{j}{4^{\nu}}, \frac{j+1}{4^{\nu}}\right)=\frac{j}{4^{\nu}}+\frac{1}{4^{\nu}} S_{j}^{\nu},
$$

where each $S_{j}^{\nu} \in\{A, B\}$. It follows that

$$
m\left(B \cap\left[\frac{j}{4^{\nu}}, \frac{j+1}{4^{\nu}}\right)\right)=\frac{1}{4^{\nu}} m\left(S_{j}^{\nu}\right)=\frac{1}{2 \cdot 4^{\nu}} .
$$

Lebesgue point ideas (see, for example, [10, Theorem 7.10]) imply that the sequence of functions $\left(f_{N}\right)_{N \in \mathbb{N}}$ on $[0,1]$ given by

$$
f_{N}(t):=\sum_{j=0}^{N-1}\left(N \int_{j / N}^{(j+1) / N} f d m\right) \chi_{[j / N,(j+1) / N)}(t)
$$

converges to $f(t) \in\{0,1\}$ for almost all $t \in[0,1]$. However, for each $t \in[0,1)$ and for all $\nu \in \mathbb{N}$, letting $N:=4^{\nu}$ gives

$$
\begin{aligned}
f_{N}(t) & =\sum_{j=0}^{N-1} N m\left(B \cap\left[\frac{j}{N}, \frac{j+1}{N}\right)\right) \chi_{[j / N,(j+1) / N)}(t) \\
& =\frac{1}{2 N} N \sum_{j=0}^{N-1} \chi_{[j / N,(j+1) / N)}(t)=\frac{1}{2} .
\end{aligned}
$$

This contradicts the fact that $\left(f_{N}\right)_{N \in \mathbb{N}}$ converges to a $\{0,1\}$-valued function.

Our main result shows how Alspach's map, in conjunction with the map $\Delta$, can be used to give new examples of fixed point free nonexpansive self-maps of weakly compact convex sets in $L^{1}[0,1]$. In particular, we show that every subset of $L^{1}[0,1]$ that contains the (nontrivial) intersection of an order interval and finitely many hyperplanes fails to have the 
fixed point property for nonexpansive mappings. To facilitate the proof, we introduce some notation.

Denote by $\mathcal{I}_{w}$ the linear functional on $L^{1}[0,1]$ determined by $w \in L^{\infty}[0,1]$; that is, $\mathcal{I}_{w}(f):=\int_{[0,1]} f w d m$ for all $f \in L^{1}[0,1]$. Also, define the order interval $[h, g]$ in $L^{1}[0,1]$ by

$$
[h, g]:=\left\{f \in L^{1}[0,1]: h \leq f \leq g \text { a.e. }\right\}
$$

for all $h, g \in L^{1}[0,1]$ with $h \leq g$ a.e. Given an order interval $[h, g]$ in $L^{1}[0,1]$, a natural number $n$, and bounded, measurable functions $w_{1}, \ldots, w_{n}$, the $\mathbb{R}^{n}$-valued set function $\mathcal{G}: \mathcal{M} \rightarrow \mathbb{R}^{n}$ defined by

$$
\mathcal{G}(A)=\left(\int\left(g \chi_{A}+h \chi_{[0,1] \backslash A}\right) w_{1} d m, \ldots, \int\left(g \chi_{A}+h \chi_{[0,1] \backslash A}\right) w_{n} d m\right)
$$

will be called the set function determined by the order interval $[h, g]$ and the bounded, measurable functions $w_{1}, \ldots, w_{n}$. It is useful to note that $\mathcal{G}$ is the translate of the vector-valued measure $\left(\int_{A}(g-h) w_{j} d m\right)_{j=1}^{n}$ by the fixed vector $\left(\int h w_{j} d m\right)_{j=1}^{n}$. We remark that, using Lyapunov's theorem and Lemma IX.3(c) in [4], it is straightforward to check that $\mathcal{G}(\mathcal{M})=$ $\left\{\left(\beta_{1}, \ldots, \beta_{n}\right) \in \mathbb{R}^{n}:[h, g] \cap \bigcap_{j=1}^{n} \mathcal{I}_{w_{j}}^{-1}\left(\beta_{j}\right) \neq \emptyset\right\}$.

The following theorem is an extension of [5, Theorem 3.2] that includes Alspach's example.

THEOREM 2. Let $[h, g]$ be a nontrivial order interval in $L^{1}[0,1]$; let $n \in \mathbb{N}$; and let $w_{1}, \ldots, w_{n}$ be in $L^{\infty}[0,1]$. Let $\mathcal{G}$ be the set function determined by $[h, g]$ and $w_{1}, \ldots, w_{n}$. If $\left(\alpha_{1}, \ldots, \alpha_{n}\right)$ is a point in the interior $\mathcal{G}(\mathcal{M})^{\circ}$ of the range of $\mathcal{G}$ and $K$ is a closed, bounded, convex subset of $L^{1}[0,1]$ satisfying

$$
K \supseteq[h, g] \cap \mathcal{I}_{w_{1}}^{-1}\left(\alpha_{1}\right) \cap \cdots \cap \mathcal{I}_{w_{n}}^{-1}\left(\alpha_{n}\right),
$$

then $K$ fails the fixed point property for nonexpansive mappings.

In the simpler setting where $n=1$ and $w_{1} \geq 0$, Theorem 2 becomes:

TheOREM 3. Let $\alpha \in \mathbb{R}$ and let $h$ and $g$ be functions in $L^{1}[0,1]$ satisfying: $h \leq g$ a.e., $h$ is not equivalent to $g$, and $\mathcal{I}_{w}(h)<\alpha<\mathcal{I}_{w}(g)$ for some nonnegative, bounded, measurable function $w$. Let $K$ be a nonempty, closed, bounded, convex subset of $L^{1}[0,1]$ such that

$$
K \supseteq[h, g] \cap \mathcal{I}_{w}^{-1}(\alpha) .
$$

Then $K$ fails the fixed point property for nonexpansive mappings.

Since the appearance of the set function $\mathcal{G}$ in Theorem 2 tends to complicate the appearance of the theorem, before giving its proof, we provide a brief sketch of the ideas that will be used. To begin with, consider the simpler setting in Theorem 3. Assuming that $h=0$, we can choose $c>0$ and a subset $E$ of $[0,1]$ with positive measure such that $c \chi_{E} \leq g$. For convenience, assume that $c=1$. If $f \in K$, it would be nice if we could nonexpansively 
project $f$ into $\left[0, \chi_{E}\right]$ and then use an analogue of Alspach's map on $E$ to get a fixed point free map from $K$ into itself. The map $R(f)=|f| \wedge \chi_{E}$ is a nonexpansive retract of $K$ onto $\left[0, \chi_{E}\right]$ but, unfortunately, $R(f)$ may fail to lie in an analogue of Alspach's set $C_{0}$ or even in the set $K$.

In order to account for these failures, we take an analogue $\widetilde{T}$ of Alspach's map and an analogue $\widetilde{\Delta}$ of the map $\Delta$ defined on functions that are measurable with respect to a certain $\sigma$-algebra $\mathcal{A}$ of subsets of $E$. We then find that $\int_{E} \widetilde{T} \widetilde{\Delta} E^{\mathcal{A}} R(f) w d m=\frac{1}{2} \int_{E} w d m$ where $E^{\mathcal{A}}$ is the conditional expectation operator with respect to $\mathcal{A}$. However, the value $\frac{1}{2} \int_{E} w d m$ may fail to equal $\alpha$ and we want a map whose range lies in $[0, g] \cap \mathcal{I}_{w}^{-1}(\alpha)$ and, hence, in $K$. In order to modify the map $\widetilde{T} \widetilde{\Delta} E^{\mathcal{A}} R$ so that its range is in $[0, g] \cap \mathcal{I}_{w}^{-1}(\alpha)$, we take the set $E$ small enough so that $\frac{1}{2} \int_{E} w d m<\alpha$ and $\int_{[0,1] \backslash E} g w d m>\alpha-\frac{1}{2} \int_{E} w d m$. We can then choose a measurable set $G \subseteq[0,1] \backslash E$ so that $\int_{G} g w d m=\alpha-\frac{1}{2} \int_{E} w d m$, the difference between the value $\alpha$ that we want and the value $\frac{1}{2} \int_{E} w d m$ that the integration provided. The map $U: K \rightarrow[0, g] \cap \mathcal{I}_{w}^{-1}(\alpha)$ defined by $U(f)=g \chi_{G}+\widetilde{T} \widetilde{\Delta} E^{\mathcal{A}} R(f)$ will then be a nonexpansive map of $K$ into itself and we will see that if $U$ has a fixed point, so will $T \Delta$, a contradiction to Lemma 1 . Thus $K$ will fail to have the fixed point property.

For the general case when $n>1$, the set function $\mathcal{G}$, in conjunction with Lyapunov's theorem, is used to find a set $E$ and then a single set $G$ that simultaneously makes up the shortfalls $\alpha_{j}-\frac{1}{2} \int_{E} w_{j} d m, j=1, \ldots, n$, needed to ensure that the resulting map sends $K$ into $K$.

Proof of Theorem 2. By translating by $-h$, relabeling $K-h$ as $K, g-h$ as $g$ and $\alpha_{j}-\mathcal{I}_{w_{j}}(h)$ as $\alpha_{j}$ for $j=1, \ldots, n$, there is no loss of generality in assuming that $h=0$ and $g \geq 0$ a.e. with $g$ nontrivial. Then $\mathcal{G}: \mathcal{M} \rightarrow \mathbb{R}^{n}$ is the $m$-continuous, countably additive vector measure defined by $\mathcal{G}(A)=$ $\left(\int_{A} g w_{1} d m, \ldots, \int_{A} g w_{n} d m\right)$. Since $\left(\alpha_{1}, \ldots, \alpha_{n}\right)$ is in the range of $\mathcal{G}$,

$$
K \supseteq[0, g] \cap \mathcal{I}_{w_{1}}^{-1}\left(\alpha_{1}\right) \cap \cdots \cap \mathcal{I}_{w_{n}}^{-1}\left(\alpha_{n}\right) \neq \emptyset .
$$

Moreover, since $\left(\alpha_{1}, \ldots, \alpha_{n}\right)$ lies in the interior of the range of $\mathcal{G}$, it is easy to see that the set $\left\{w_{1}, \ldots, w_{n}\right\}$ is a linearly independent set of functions in $L^{\infty}[0,1]$. Thus $w_{1} \neq 0$. In fact, $w_{1}$ is not identically 0 on the set $[g>0]$. Indeed, if $w_{1}$ vanishes almost everywhere on $[g>0]$, then, for every $A \in \mathcal{M}$, $\mathcal{G}(A)=\left(0, \int_{A} g w_{2} d m, \ldots, \int_{A} g w_{n} d m\right)$ and $\mathcal{G}(\mathcal{M})^{\circ}=\emptyset$, a contradiction.

Let $E_{1}^{\prime}$ be a measurable subset of $[g>0]$ such that $m\left(E_{1}^{\prime}\right)>0$ and, without loss of generality, $w_{1}(t)>0$ for each $t \in E_{1}^{\prime}$. Then there exists a real number $c>0$ and a measurable set $E_{1}^{\prime \prime} \subseteq E_{1}^{\prime}$ with $m\left(E_{1}^{\prime \prime}\right)>0$ such that $g \geq c \chi_{E_{1}^{\prime \prime}}$. By rescaling $K$ and $\alpha_{1}, \ldots, \alpha_{n}$ by $1 / c$, there is no loss of generality in assuming that $c=1$. Let $E_{1}$ denote this rescaled $E_{1}^{\prime \prime}$. Thus $E_{1}$ is a subset of $[g \geq 1]$ with $m\left(E_{1}\right)>0$ and $w_{1}(t)>0$ for each $t \in E_{1}$. 
Now consider $w_{2}$. If $w_{2}$ vanishes almost everywhere on $E_{1}$, set $E_{2}:=E_{1}$. Otherwise there exists a subset $E_{2}$ of $E_{1}$ with $m\left(E_{2}\right)>0$ and either $w_{2}(t)>0$ for each $t \in E_{2}$ or $w_{2}(t)<0$ for each $t \in E_{2}$. Repeating this process for $j=3, \ldots, n$ gives a decreasing sequence of sets $E_{1} \supseteq \cdots \supseteq E_{n}$ such that, if $E^{\prime}:=E_{n}$, then $w_{1}(t)>0$ for each $t \in E^{\prime}$ and, for each $j=2, \ldots, n$, one of the following holds: $w_{j}(t)=0$ for each $t \in E^{\prime} ; w_{j}(t)>0$ for each $t \in E^{\prime}$; or $w_{j}(t)<0$ for each $t \in E^{\prime}$.

Let $J:=\left\{j \in\{1, \ldots, n\}: w_{j}(t)>0\right.$ for each $t \in E^{\prime}$ or $w_{j}(t)<0$ for each $\left.t \in E^{\prime}\right\}$. Obviously $1 \in J$.

Since $\left(\alpha_{1}, \ldots, \alpha_{n}\right) \in \mathcal{G}(\mathcal{M})^{\circ}$, there exists $0<\delta<1 / 2$ so that the closed ball $B\left(\left(\alpha_{1}, \ldots, \alpha_{n}\right), \delta\right) \subseteq \mathcal{G}(\mathcal{M})$. By compactness of the sphere $S:=$ $S\left(\left(\alpha_{1}, \ldots, \alpha_{n}\right), \delta / 2\right)$, there exists a finite set $\left\{y_{1}, \ldots, y_{N}\right\}$ in $S$ such that $\left\{y_{1}, \ldots, y_{N}\right\}$ is a $\delta^{2} / 4$-net for $S$. Since this set lies in the range of $\mathcal{G}$, for each $i=1, \ldots, N$ there exists a measurable set $A_{i}$ in $[0,1]$ with $y_{i}=\mathcal{G}\left(A_{i}\right)$.

Let $M:=\sup _{j=1, \ldots, n}\left\|w_{j}\right\|_{\infty}$. Choose a measurable subset $E$ of $E^{\prime}$ satisfying $0<m(E)<\delta / 2 M \sqrt{n}$ and $|\mathcal{G}|(E)<\delta^{2} / 4$. This is possible since the measure space is nonatomic and the variation $|\mathcal{G}|$ of the vector measure $\mathcal{G}$ is $m$-continuous.

For $i=1, \ldots, N$, define $z_{i}=\mathcal{G}\left(A_{i} \backslash E\right)$ in $\left(\mathbb{R}^{n},\|\cdot\|_{2}\right)$. Then, for $i=$ $1, \ldots, N$,

$$
\left\|y_{i}-z_{i}\right\|_{2}=\left\|\mathcal{G}\left(A_{i}\right)-\mathcal{G}\left(A_{i} \backslash E\right)\right\|_{2}=\left\|\mathcal{G}\left(A_{i} \cap E\right)\right\|_{2} \leq|\mathcal{G}|(E)<\delta^{2} / 4 .
$$

A quick application of the triangle inequality shows that

$$
S=S\left(\left(\alpha_{1}, \ldots, \alpha_{n}\right), \delta / 2\right) \subseteq \bigcup_{i=1}^{N}\left(z_{i}+B\left((0, \ldots, 0), \delta^{2} / 2\right)\right) .
$$

Claim. $B\left(\left(\alpha_{1}, \ldots, \alpha_{n}\right), \delta / 4\right) \subseteq \operatorname{co}\left\{z_{1}, \ldots, z_{N}\right\}$.

An argument proving this claim can be found in [7, Lemma 2.2]. Since these notes are unpublished, we include a short proof. Taking convex hulls in (1) yields

$$
B\left(\left(\alpha_{1}, \ldots, \alpha_{n}\right), \delta / 2\right) \subseteq \operatorname{co}\left\{z_{1}, \ldots, z_{N}\right\}+B\left((0, \ldots, 0), \delta^{2} / 2\right)
$$

or, equivalently, with $\vec{\alpha}:=\left(\alpha_{1}, \ldots, \alpha_{n}\right)$ and $\overrightarrow{0}:=(0, \ldots, 0)$,

$$
B(\overrightarrow{0}, \delta / 2) \subseteq \operatorname{co}\left\{z_{1}-\vec{\alpha}, \ldots, z_{N}-\vec{\alpha}\right\}+\delta \cdot B(\overrightarrow{0}, \delta / 2) .
$$

Then, if $u_{0} \in B(\overrightarrow{0}, \delta / 2)$, there exist $b_{k} \in \operatorname{co}\left\{z_{1}-\vec{\alpha}, \ldots, z_{N}-\vec{\alpha}\right\}$ and $u_{k} \in$ $B(\overrightarrow{0}, \delta / 2)$ such that

$$
u_{k-1}=b_{k}+\delta u_{k}
$$

for all $k \in \mathbb{N}$. Note that $\delta^{k} u_{k} \rightarrow 0$,

$$
\frac{1-\delta}{1-\delta^{l}} \sum_{k=1}^{l} \delta^{k-1} b_{k}=\sum_{k=1}^{l} \frac{\delta^{k-1}}{1+\delta+\cdots+\delta^{l-1}} b_{k} \in \operatorname{co}\left\{z_{1}-\vec{\alpha}, \ldots, z_{N}-\vec{\alpha}\right\},
$$


and $\operatorname{co}\left\{z_{1}-\vec{\alpha}, \ldots, z_{N}-\vec{\alpha}\right\}$ is closed. It follows that

$$
u_{0}=\sum_{k=1}^{\infty} \delta^{k-1} b_{k} \in \frac{1}{1-\delta} \operatorname{co}\left\{z_{1}-\vec{\alpha}, \ldots, z_{N}-\vec{\alpha}\right\} .
$$

Therefore $(1-\delta) B(\overrightarrow{0}, \delta / 2) \subseteq \operatorname{co}\left\{z_{1}-\vec{\alpha}, \ldots, z_{N}-\vec{\alpha}\right\}$ or, equivalently,

$$
B(\vec{\alpha},(1-\delta) \delta / 2) \subseteq \operatorname{co}\left\{z_{1}, \ldots, z_{N}\right\} .
$$

Since $0<\delta<1 / 2$, the Claim follows.

Note also that the vector $\left(\alpha_{1}-\frac{1}{2} \int_{E} w_{1} d m, \ldots, \alpha_{n}-\frac{1}{2} \int_{E} w_{n} d m\right)$ is in $B\left(\left(\alpha_{1}, \ldots, \alpha_{n}\right), \delta / 4\right)$. This follows from the choice of $E$ and

$$
\left\|\left(\frac{1}{2} \int_{E} w_{1} d m, \ldots, \frac{1}{2} \int_{E} w_{n} d m\right)\right\|_{2} \leq \frac{1}{2} M \sqrt{n} m(E) \leq \frac{\delta}{4} .
$$

Then, by the Claim, the choice of the $z_{j}$ 's in the range $\mathcal{G}\left(\mathcal{M}_{[0,1] \backslash E}\right)$ of the nonatomic, countably additive vector measure $\mathcal{G}$, and Lyapunov's theorem $[4$, p. 264], it follows that

$$
\left(\alpha_{1}-\frac{1}{2} \int_{E} w_{1} d m, \ldots, \alpha_{n}-\frac{1}{2} \int_{E} w_{n} d m\right) \in \mathcal{G}\left(\mathcal{M}_{[0,1] \backslash E}\right)
$$

where $\mathcal{M}_{[0,1] \backslash E}$ is the $\sigma$-algebra of Lebesgue measurable subsets of $[0,1] \backslash E$. Therefore there exists a measurable set $G \subseteq[0,1] \backslash E$ such that

$$
\mathcal{G}(G)=\left(\alpha_{1}-\frac{1}{2} \int_{E} w_{1} d m, \ldots, \alpha_{n}-\frac{1}{2} \int_{E} w_{n} d m\right) .
$$

Now, consider the sets $E$ and $G$ as chosen above, and let $\nu_{j}(A):=$ $\int_{A} w_{j} d m$ for $j=1, \ldots, n$.

Claim. There exist measurable subsets $\left(E_{i, k}\right)_{i \in \mathbb{N} \cup\{0\}, k=1, \ldots, 2^{i}}$ of $E$ which are "dyadic sets" for the measures $m$ and $\nu_{j}$ for all $j=1, \ldots, n$; that is, $E_{0,1}=E, E_{i, k}=E_{i+1,2 k-1} \cup E_{i+1,2 k}, E_{i, k} \cap E_{i, l}=\emptyset$ if $k \neq l, m\left(E_{i, k}\right)=$ $m(E) / 2^{i}$, and $\nu_{j}\left(E_{i, k}\right)=\nu_{j}(E) / 2^{i}$ for $j=1, \ldots, n$.

To prove the Claim, define a vector measure $\mathcal{F}: \mathcal{M}_{E} \rightarrow \mathbb{R}^{n+1}$ by

$$
\mathcal{F}(A):=\left(m(A), \nu_{1}(A), \ldots, \nu_{n}(A)\right)
$$

for each $A \in \mathcal{M}_{E}$. Lyapunov's theorem implies that $\mathcal{F}\left(\mathcal{M}_{E}\right)$ is a convex subset of $\mathbb{R}^{n+1}$. Therefore, since

$$
\mathcal{F}(\emptyset)=(0, \ldots, 0) \quad \text { and } \quad \mathcal{F}(E)=\left(m(E), \nu_{1}(E), \ldots, \nu_{n}(E)\right),
$$

there is a measurable subset $E_{1,1}$ of $E$ such that

$$
\mathcal{F}\left(E_{1,1}\right)=\left(\frac{1}{2} m(E), \frac{1}{2} \nu_{1}(E), \ldots, \frac{1}{2} \nu_{n}(E)\right) .
$$

Letting $E_{1,2}=E \backslash E_{1,1}$, we easily see that

$$
\mathcal{F}\left(E_{1,2}\right)=\left(\frac{1}{2} m(E), \frac{1}{2} \nu_{1}(E), \ldots, \frac{1}{2} \nu_{n}(E)\right) .
$$


Using Lyapunov's theorem again on the sets $E_{1,1}$ and $E_{1,2}$, we get pairwise disjoint sets $E_{2,1}, E_{2,2}, E_{2,3}, E_{2,4}$ such that

$$
E_{1,1}=E_{2,1} \cup E_{2,2}, \quad E_{1,2}=E_{2,3} \cup E_{2,4},
$$

and

$$
\mathcal{F}\left(E_{2, l}\right)=\left(\frac{1}{4} m(E), \frac{1}{4} \nu_{1}(E), \ldots, \frac{1}{4} \nu_{n}(E)\right)
$$

for all $l=1,2,3,4$.

Continuing this process inductively completes the proof of the Claim.

Let $\mathcal{A}$ denote the $\sigma$-algebra of subsets of $E$ generated by the sets $\left(E_{i, k}\right)$. Then, with the measure $\mu$ defined on the $\mathcal{A}$-measurable subsets of $E$ by $\mu:=m / m(E)=\nu_{j} / \nu_{j}(E)$ for $j \in J$, it follows that the Banach space $L^{1}(E, \mathcal{A}, \mu)$ is isometrically isomorphic to $L^{1}([0,1], m)=L^{1}[0,1]$ via the mapping $Z$ defined as follows:

$$
Z\left(\chi_{E_{i, k}}\right):=\chi_{\left[(k-1) / 2^{i}, k / 2^{i}\right)}
$$

for each $\chi_{E_{i, k}}$. The mapping $Z$ is extended to the linear span $L$ of the functions $\chi_{E_{i, k}}$ in the usual way. Of course, $Z$ is an isometry on $L$. Finally, since $L$ is dense in $L^{1}(E, \mathcal{A}, \mu)$ and $Z(L)$ is dense in $L^{1}[0,1], Z$ extends to a linear isometry from $L^{1}(E, \mathcal{A}, \mu)$ onto $L^{1}[0,1]$.

Analogues of Alspach's map $T$ and the map $\Delta$ will now be defined directly on $E$ using the dyadic sets $E_{i, k}$. For an $\mathcal{A}$-measurable function $f=\sum_{k=1}^{2^{i}} \alpha_{k} \chi_{E_{i, k}}$ in $\left[0, \chi_{E}\right]$, define

$$
\widetilde{\Delta}(f)=\sum_{k=1}^{2^{i}}\left(\alpha_{k} \chi_{E_{i+1, k}}+\left(1-\alpha_{k}\right) \chi_{E_{i+1,2^{i}+k}}\right) .
$$

Since the functions of the form $\sum_{k=1}^{2^{i}} \alpha_{k} \chi_{E_{i, k}}$ are dense in $L^{1}(E, \mathcal{A}, \mu)$, the definition can be extended to define a map $\widetilde{\Delta}:\left[0, \chi_{E}\right] \cap L^{1}(E, \mathcal{A}, \mu) \rightarrow$ $\left[0, \chi_{E}\right] \cap L^{1}(E, \mathcal{A}, \mu)$. Also, since $\Delta$ and $Z$ are isometries and $\widetilde{\Delta}=Z^{-1} \Delta Z$, $\widetilde{\Delta}$ is an $L^{1}(E, \mathcal{A}, \mu)$-isometry on $\left[0, \chi_{E}\right] \cap L^{1}(E, \mathcal{A}, \mu)$.

An easy computation shows that, if $f$ is an $\mathcal{A}$-measurable function in $\left[0, \chi_{E}\right]$, then

$$
\int_{E} \widetilde{\Delta}(f) w_{j} d m=\frac{1}{2} \nu_{j}(E)=\frac{1}{2} \int_{E} w_{j} d m
$$

for $j=1, \ldots, n$. Indeed, it suffices to check the equality for functions $f$ of the form $\sum_{k=1}^{2^{i}} \alpha_{k} \chi_{E_{i, k}}$. So, if $f=\sum_{k=1}^{2^{i}} \alpha_{k} \chi_{E_{i, k}}$ is in the order interval $\left[0, \chi_{E}\right]$, then 


$$
\begin{aligned}
\int_{E} \widetilde{\Delta}(f) w_{j} d m & =\sum_{k=1}^{2^{i}}\left(\alpha_{k} \nu_{j}\left(E_{i+1, k}\right)+\left(1-\alpha_{k}\right) \nu_{j}\left(E_{i+1,2^{i}+k}\right)\right) \\
& =\sum_{k=1}^{2^{i}}\left(\alpha_{k} \frac{1}{2^{i+1}} \nu_{j}(E)+\left(1-\alpha_{k}\right) \frac{1}{2^{i+1}} \nu_{j}(E)\right) \\
& =\sum_{k=1}^{2^{i}} \frac{1}{2^{i+1}} \nu_{j}(E)=\frac{1}{2} \nu_{j}(E) .
\end{aligned}
$$

Similarly, an analogue of Alspach's map can be defined directly on $E$. With $f=\sum_{k=1}^{2^{i}} \alpha_{k} \chi_{E_{i, k}}$, define

$$
\widetilde{T}(f)=\sum_{k=1}^{2^{i}}\left(\left(2 \alpha_{k} \wedge 1\right) \chi_{E_{i+1, k}}+\left(\left(2 \alpha_{k}-1\right) \vee 0\right) \chi_{E_{i+1,2^{i}+k}}\right) \text {. }
$$

As before, $\widetilde{T}$ extends to a map from $\left[0, \chi_{E}\right] \cap L^{1}(E, \mathcal{A}, \mu)$ into itself. Using the Alspach map $T$ on $L^{1}[0,1]$ and the fact that $\widetilde{T}=Z^{-1} T Z$, we see that $\widetilde{T}$ is an isometry sending $\left[0, \chi_{E}\right] \cap L^{1}(E, \mathcal{A}, \mu)$ into itself. Note also that, for $f=\sum_{k=1}^{2^{i}} \alpha_{k} \chi_{E_{i, k}}$ in $\left[0, \chi_{E}\right] \cap L^{1}(E, \mathcal{A}, \mu)$ and $j=1, \ldots, n$,

$$
\begin{aligned}
\int_{E} \widetilde{T}(f) & w_{j} d m \\
& =\sum_{k=1}^{2^{i}}\left(\left(2 \alpha_{k} \wedge 1\right) \nu_{j}\left(E_{i+1, k}\right)+\left(\left(2 \alpha_{k}-1\right) \vee 0\right) \nu_{j}\left(E_{i+1,2^{i}+k}\right)\right) \\
& =\sum_{k=1}^{2^{i}}\left(\left(2 \alpha_{k} \wedge 1\right)+\left(\left(2 \alpha_{k}-1\right) \vee 0\right)\right) \frac{1}{2^{i+1}} \nu_{j}(E) \\
& =\sum_{k=1}^{2^{i}} \alpha_{k} \frac{1}{2^{i}} \nu_{j}(E)=\sum_{k=1}^{2^{i}} \alpha_{k} \nu_{j}\left(E_{i, k}\right)=\int_{E} f w_{j} d m
\end{aligned}
$$

By the density of such $f$ in $\left[0, \chi_{E}\right] \cap L^{1}(E, \mathcal{A}, \mu)$, this equality holds for all $f$ in $\left[0, \chi_{E}\right] \cap L^{1}(E, \mathcal{A}, \mu)$.

In order to define a nonexpansive self-map of $K$ without a fixed point, it is useful to define a few more preliminary maps. Let $E^{\mathcal{A}}$ be the conditional expectation operator with respect to the $\sigma$-algebra $\mathcal{A}$ and let $R: K \rightarrow\left[0, \chi_{E}\right]$ be a restriction map defined by

$$
R(f):=|f| \wedge \chi_{E} \quad \text { for all } f \in K .
$$

Then $E^{\mathcal{A}}$ is a nonexpansive map from $L^{1}\left(E, \mathcal{M}_{E}, \mu\right)$ onto $L^{1}(E, \mathcal{A}, \mu)$. (For a proof of this, see [4, p. 122].) The map $R$ is $L^{1}[0,1]$-nonexpansive and $R$ equals the identity on $\left[0, \chi_{E}\right] \cap \mathcal{I}_{w_{1}}^{-1}\left(\alpha_{1}\right) \cap \cdots \cap \mathcal{I}_{w_{n}}^{-1}\left(\alpha_{n}\right) \subseteq K$. Note that, 
for any $f \in K,(3)$ and (4) imply that

$$
\widetilde{T} \widetilde{\Delta} E^{\mathcal{A}} R(f) \in\left[0, \chi_{E}\right] \cap L^{1}(E, \mathcal{A}, \mu) \cap \bigcap_{j=1}^{n} \mathcal{I}_{w_{j}}^{-1}\left(\frac{1}{2} \int_{E} w_{j} d m\right) .
$$

It is now time to reconsider the set $H:=[0, g] \cap \mathcal{I}_{w_{1}}^{-1}\left(\alpha_{1}\right) \cap \cdots \cap \mathcal{I}_{w_{n}}^{-1}\left(\alpha_{n}\right)$ $\subseteq K$. Note that the function $\widetilde{T} \widetilde{\Delta} E^{\mathcal{A}} R(f)$ lies in the order interval $[0, g]$, has support in $E$, but there is no guarantee that it lies on any of the hyperplanes $\mathcal{I}_{w_{j}}^{-1}\left(\alpha_{j}\right)$. Thus, for any $f \in K, \widetilde{T} \widetilde{\Delta} E^{\mathcal{A}} R(f)$ may fail to be a member of $H$. Recall also the choice of the set $G$ defined in (2), and define the function $\phi:=g \chi_{G}$ and the set $H_{0} \subseteq H$ by

$$
\begin{aligned}
H_{0}:=\left\{\phi+u: u \in\left[0, \chi_{E}\right], u \text { is } \mathcal{A}\right. \text {-measurable, } \\
\left.\quad \text { and } \int_{E} u w_{j} d m=\frac{1}{2} \int_{E} w_{j} d m \text { for } j=1, \ldots, n\right\} .
\end{aligned}
$$

It is easy to check that $H_{0}=\phi+Z^{-1}\left(C_{0}\right)$. Indeed, if $f \in C_{0}$ then $f \in C=$ $\left[0, \chi_{[0,1]}\right]$, and so $Z^{-1}(f)$ is an $\mathcal{A}$-measurable function in $\left[0, \chi_{E}\right]$. Furthermore, for $j=1, \ldots, n$,

$$
\begin{aligned}
\int_{E} Z^{-1} & (f) w_{j} d m \\
& =\int_{E} Z^{-1}(f) d \nu_{j}=\nu_{j}(E) \int_{E} Z^{-1}(f) d \mu \\
& =\nu_{j}(E) \int_{[0,1]} f d m \quad \text { since } Z \text { is an isometry and } f, Z^{-1}(f) \geq 0 \\
& =\nu_{j}(E) \cdot \frac{1}{2} \quad \text { since } f \in C_{0} \\
& =\frac{1}{2} \int_{E} w_{j} d m .
\end{aligned}
$$

So, $\phi+Z^{-1}\left(C_{0}\right) \subseteq H_{0}$. The reverse set inclusion is proven similarly. In particular, $H_{0}$ is nonempty.

Moreover, for any $u \in Z^{-1}\left(C_{0}\right)$, the maps $u$ and $\phi=g \chi_{G}$ are disjointly supported. Thus

$$
0 \leq \phi+u \leq g \chi_{[0,1] \backslash E}+g \chi_{E}=g
$$

and, by the definition of $G$ in equation (2),

$$
\mathcal{I}_{w_{j}}(\phi+u)=\int_{[0,1]} \phi w_{j} d m+\int_{[0,1]} u w_{j} d m=\int_{G} g w_{j} d m+\frac{1}{2} \int_{E} w_{j} d m=\alpha_{j}
$$

for $j=1, \ldots, n$. Thus $H_{0} \subseteq H \subseteq K$. 
Finally, define the mapping $U: K \rightarrow H_{0} \subseteq K$ by

$$
U(f):=\phi+\widetilde{T} \widetilde{\Delta} E^{\mathcal{A}} R(f) \quad \text { for all } f \in K
$$

By (5), $U$ maps into $H_{0}$. It remains to show that $U$ is a nonexpansive map without a fixed point.

In order to see that $U$ is nonexpansive, take $f_{1}, f_{2} \in K$. Then

$$
\begin{aligned}
& \left\|U\left(f_{1}\right)-U\left(f_{2}\right)\right\|_{L^{1}[0,1]} \\
& =\int_{[0,1]}\left|\widetilde{T} \widetilde{\Delta} E^{\mathcal{A}} R\left(f_{1}\right)-\widetilde{T} \widetilde{\Delta} E^{\mathcal{A}} R\left(f_{2}\right)\right| d m \\
& =m(E) \int_{E}\left|\widetilde{T} \widetilde{\Delta} E^{\mathcal{A}} R\left(f_{1}\right)-\widetilde{T} \widetilde{\Delta} E^{\mathcal{A}} R\left(f_{2}\right)\right| d \mu \\
& =m(E) \int_{E}\left|E^{\mathcal{A}} R\left(f_{1}\right)-E^{\mathcal{A}} R\left(f_{2}\right)\right| d \mu \quad \text { since } \widetilde{T}, \widetilde{\Delta} \text { are } L^{1}(E, \mathcal{A}, \mu) \text {-isometries } \\
& \leq m(E) \int_{E}\left|R\left(f_{1}\right)-R\left(f_{2}\right)\right| d \mu \quad \text { since } E^{\mathcal{A}} \text { is nonexpansive } \\
& =\int_{E}\left|R\left(f_{1}\right)-R\left(f_{2}\right)\right| d m=\left\|R\left(f_{1}\right)-R\left(f_{2}\right)\right\|_{L^{1}[0,1]} \\
& \leq\left\|f_{1}-f_{2}\right\|_{L^{1}[0,1]} \quad \text { since } R \text { is nonexpansive. }
\end{aligned}
$$

It follows that $U: K \rightarrow K$ is $L^{1}[0,1]$-nonexpansive.

To see that $U$ is fixed point free on $K$, suppose, for the sake of a contradiction, that $f \in K$ is a fixed point of $U$. Then

$$
f=U(f)=\phi+\widetilde{T} \widetilde{\Delta} E^{\mathcal{A}} R(f) .
$$

But this implies that $f=U(f) \in H_{0}$. Thus $f=g \chi_{G}+u$, where $u=$ $\widetilde{T} \widetilde{\Delta} E^{\mathcal{A}} R(f)$ is an $\mathcal{A}$-measurable function in $\left[0, \chi_{E}\right]$ and $G$ and $E$ are disjoint. Consequently, $R(f)=\left(g \chi_{G}+u\right) \wedge \chi_{E}=u$ and, since $u$ is $\mathcal{A}$-measurable, $u=E^{\mathcal{A}} R(f)$. Hence,

$$
E^{\mathcal{A}} R(f)=R(f)=u=\widetilde{T} \widetilde{\Delta} E^{\mathcal{A}} R(f) .
$$

Thus the function $E^{\mathcal{A}} R(f)$ is a fixed point of $\widetilde{T} \widetilde{\Delta}$. Since $\widetilde{T}=Z^{-1} T Z$ and $\widetilde{\Delta}=Z^{-1} \Delta Z$, this implies that

$$
Z^{-1} T \Delta Z\left(E^{\mathcal{A}} R(f)\right)=E^{\mathcal{A}} R(f),
$$

or

$$
T \Delta\left(Z E^{\mathcal{A}} R(f)\right)=Z E^{\mathcal{A}} R(f) .
$$

Since $Z E^{\mathcal{A}} R(f)$ lies in $C$ for each $f$ in $K$, this contradicts the conclusion of Lemma 1 that $T \Delta$ is fixed point free on $C$, and the proof is complete.

REMARK. Note that the proof of Theorem 2 still works if we everywhere replace $m$ by any nontrivial, finite, positive, purely nonatomic measure $\mu$ on a measurable space. 
It should also be noted that some restriction on the location of the point $\left(\alpha_{1}, \ldots, \alpha_{n}\right)$ in Theorem 2 is necessary, although the condition used in Theorem 2 , that the point lies in the interior of the range of the set function $\mathcal{G}$, is not the most general possible. It is easy to give examples in the setting of Theorem 3 where $K$ is a singleton and hence has the fixed point property if $\alpha$ were equal to either $\mathcal{I}_{w}(h)$ or $\mathcal{I}_{w}(g)$.

It is perhaps more instructive to consider examples when $n=2$ in Theorem 2. If $h \equiv 0, g \equiv 1, w_{1}(t)=t$, and $w_{2}(t)=1-t$, the set function $\mathcal{G}$ is defined by $\mathcal{G}(A)=\left(\int_{A} w_{1} d m, \int_{A} w_{2} d m\right)$ and its range $\mathcal{G}(\mathcal{M})$ is the region in the plane bounded by the graphs of $y=\sqrt{2 x}-x$ and $x=\sqrt{2 y}-y$. If $\left(\alpha_{1}, \alpha_{2}\right)$ is a point on the boundary of $\mathcal{G}(\mathcal{M})$, the set $[h, g] \cap \mathcal{I}_{w_{1}}^{-1}\left(\alpha_{1}\right) \cap \mathcal{I}_{w_{2}}^{-1}\left(\alpha_{2}\right)$ is a singleton. As a second example, with $h \equiv 0, g \equiv 1, w_{1}(t)=\chi_{[0,1 / 2]}$, and $w_{2}(t)=\chi_{[1 / 2,1]}$, it is easy to check that $\mathcal{G}(\mathcal{M})$ is the square $[0,1 / 2] \times[0,1 / 2]$ and the sets $[h, g] \cap \mathcal{I}_{w_{1}}^{-1}\left(\alpha_{1}\right) \cap \mathcal{I}_{w_{2}}^{-1}\left(\alpha_{2}\right)$ fail the fixed point property for all points $\left(\alpha_{1}, \alpha_{2}\right)$ in the closed square other than the four corner points. It is therefore reasonable to ask if the interior point hypothesis in Theorem 2 can be relaxed so that the conclusion of the theorem holds whenever the point $\left(\alpha_{1}, \ldots, \alpha_{n}\right)$ is not an extreme point of $\mathcal{G}(\mathcal{M})$.

We finish with some consequences of Theorem 2.

Corollary 4. Let $X$ be a subspace of $\left(L^{1}[0,1],\|\cdot\|_{1}\right)$ of codimension $n \in \mathbb{N}$. Let $g \in L^{1}[0,1]$ with $g \geq 0$ and $g \neq 0$, and let $K$ be a closed, bounded, convex subset of $X$ satisfying $K \supseteq[-g, g] \cap X$. Then $K$ fails the fixed point property for nonexpansive mappings.

Proof. By our hypotheses, there exist linearly independent functions $w_{1}, \ldots, w_{n} \in L^{\infty}[0,1]$ such that

$$
X=\bigcap_{j=1}^{n} \mathcal{I}_{w_{j}}^{-1}(0)=\left\{f \in L^{1}[0,1]: \int_{[0,1]} f w_{j} d m=0 \text { for all } j=1, \ldots, n\right\} .
$$

First, consider the special case where $g>0$ a.e. on $[0,1]$. As in Theorem 2, consider the set function $\mathcal{G}: \mathcal{M} \rightarrow \mathbb{R}^{n}$ given by

$$
\mathcal{G}(A):=\left(\int_{A} g w_{j} d m-\int_{A^{\mathrm{c}}} g w_{j} d m\right)_{j=1}^{n} \quad \text { for all } A \in \mathcal{M} .
$$

Claim. The linear span of $\mathcal{G}(\mathcal{M})$ is $\mathbb{R}^{n}$.

To prove the Claim, assume the contrary. Then there exist $a_{1}, \ldots, a_{n}$ $\in \mathbb{R}$, not all zero, with $\mathcal{G}(\mathcal{M}) \subseteq\left\{\left(x_{1}, \ldots, x_{n}\right) \in \mathbb{R}^{n}: a_{1} x_{1}+\cdots+a_{n} x_{n}=0\right\}$. Thus, for all $A \in \mathcal{M}$,

$$
\sum_{j=1}^{n} a_{j}\left(\int_{A} g w_{j} d m-\int_{A^{\mathrm{c}}} g w_{j} d m\right)=0
$$


therefore

$$
2 \sum_{j=1}^{n} a_{j} \int_{A} g w_{j} d m=\sum_{j=1}^{n} a_{j} \int_{[0,1]} g w_{j} d m=: y
$$

Letting $A=\emptyset$ shows that $y=0$, and so for all $A \in \mathcal{M}, \int_{A}\left(\sum_{j=1}^{n} a_{j} g w_{j}\right) d m$ $=0$. Hence, $g \sum_{j=1}^{n} a_{j} w_{j}=0$ almost everywhere on $[0,1]$. Since, in this special case, $g>0$ a.e., we get $\sum_{j=1}^{n} a_{j} w_{j}=0$. This contradicts the linear independence of $\left\{w_{1}, \ldots, w_{n}\right\}$ and the Claim is proven.

Next, note that $\mathcal{G}([0,1])=-\mathcal{G}(\emptyset)$. Since $\mathcal{G}$ is a translate of a vector measure, by Lyapunov's theorem, $\overrightarrow{0}=(\mathcal{G}(\emptyset)+\mathcal{G}([0,1])) / 2 \in \mathcal{G}(\mathcal{M})$. Further, for all $\gamma=\mathcal{G}(A) \in \mathcal{G}(\mathcal{M})$, it follows that $-\gamma=\mathcal{G}\left(A^{\mathrm{c}}\right) \in \mathcal{G}(\mathcal{M})$. Hence, as observed by E. Bolker $[2], \mathcal{G}(\mathcal{M})$ is symmetric about $\overrightarrow{0}$, and so, by the Claim, $\overrightarrow{0}$ is an internal point of $\mathcal{G}(\mathcal{M})$ in $\mathbb{R}^{n}$. (For the definition of an internal point of a set, see [9, p. 239].) Since internal points of convex sets in finite-dimensional spaces are interior points, $\overrightarrow{0}$ is an interior point of $\mathcal{G}(\mathcal{M})[9$, p. 243, Problem 44(b)]. Indeed, from the Claim, there exist $n$ linearly independent vectors $\vec{q}_{1}, \ldots, \vec{q}_{n} \in \mathcal{G}(\mathcal{M})$. Define the subset $P$ of $\mathbb{R}^{n}$ by $P:=\left\{s_{1} \vec{q}_{1}+\cdots+s_{n} \vec{q}_{n}:\left|s_{1}\right|+\cdots+\left|s_{n}\right| \leq 1\right\}$. Clearly, $P$ is a convex, balanced, absorbing subset of $\mathbb{R}^{n}$, and so the Minkowski functional $\mu_{P}$ of $P$ is a norm on $\mathbb{R}^{n}$ equivalent to the usual $\|\cdot\|_{2}$ norm. Thus, $\overrightarrow{0}$ is an interior point of $P$ and, by Lyapunov's theorem, $P \subseteq \mathcal{G}(\mathcal{M})$. Hence, $\overrightarrow{0}$ is an interior point of $\mathcal{G}(\mathcal{M})$.

Because $K \supseteq[-g, g] \cap X=[-g, g] \cap \mathcal{I}_{w_{1}}^{-1}(0) \cap \cdots \cap \mathcal{I}_{w_{n}}^{-1}(0)$, Theorem 2 implies that $K$ fails the fixed point property for nonexpansive mappings.

Finally, consider the general case: $g \geq 0$ and $g \neq 0$. Define $\Omega:=[g>0]$; note that $m(\Omega)>0$. Consider

$$
\begin{aligned}
& {[-g, g] \cap X} \\
& =\left\{f \in L^{1}[0,1]:-g \leq f \leq g \text { and } \int_{[0,1]} f w_{j} d m=0 \text { for all } j=1, \ldots, n\right\} \\
& =\left\{f \in L^{1}[0,1]:-g \leq f \leq g \text { on } \Omega, f=0 \text { on } \Omega^{\mathrm{c}}, \text { and each } \int_{\Omega} f w_{j} d m=0\right\}
\end{aligned}
$$

We will denote by $L^{1}(\Omega)$ the space of all Lebesgue integrable functions from $\Omega$ into $\mathbb{R}$. We identify $L^{1}(\Omega)$ with a subspace of $L^{1}[0,1]$ by extending each $f \in L^{1}(\Omega)$ to be identically zero on $\Omega^{\mathrm{c}}$. Note that $g \in L^{1}(\Omega)$. Then $[-g, g] \cap X=[-g, g] \cap Y$, where $Y:=\left\{f \in L^{1}(\Omega): \int_{\Omega} f w_{j} d m=0\right.$ for all $j=1, \ldots, n\}$ is a subspace of $L^{1}(\Omega)$ of codimension at most $n$. By Theorem 2 and the remark following its proof, the general case reduces to the special case proven above. 
Corollary 5. If $X$ is a subspace of $\left(L^{1}[0,1],\|\cdot\|_{1}\right)$ of codimension $n$, then $X$ contains a nonempty, weakly compact, convex set which fails the fixed point property for nonexpansive mappings.

Stated more succinctly, subspaces of codimension $n$ in $L^{1}[0,1]$ fail the weak fixed point property. This also follows from the proof of Theorem 2, which shows that all subspaces of finite codimension in $L^{1}[0,1]$ actually contain an isometric copy of $L^{1}[0,1]$. This latter fact is already known. Indeed, it follows from Theorem 1 in Section 10 of Plichko and Popov [8].

We thank Professors Bill Johnson, Mikhail Popov, and the referee for helpful suggestions concerning this paper.

\section{References}

[1] D. E. Alspach, A fixed point free nonexpansive map, Proc. Amer. Math. Soc. 82 (1981), 423-424.

[2] E. D. Bolker, A class of convex bodies, Trans. Amer. Math. Soc. 145 (1969), 323-345.

[3] J. Diestel, Sequences and Series in Banach Spaces, Springer, New York, 1984.

[4] J. Diestel and J. J. Uhl, Jr., Vector Measures, Amer. Math. Soc., Providence, RI, 1977.

[5] P. N. Dowling, C. J. Lennard and B. Turett, Some more examples of subsets of $c_{0}$ and $L^{1}[0,1]$ failing the fixed point property, in: Contemp. Math. 328, Amer. Math. Soc., 2003, 171-176.

[6] K. Goebel and W. A. Kirk, Topics in Metric Fixed Point Theory, Cambridge Univ. Press, Cambridge, 1990.

[7] H. P. Lotz, Grothendieck Ideals of Operators on Banach Spaces, Univ. of Illinois, Urbana, Il, 1973.

[8] A. M. Plichko and M. M. Popov, Symmetric function spaces on atomless probability spaces, Dissertationes Math. 306 (1990).

[9] H. Royden, Real Analysis, 3rd ed., Macmillan, New York, 1988.

[10] W. Rudin, Real and Complex Analysis, 3rd ed., McGraw-Hill, New York, 1987.

[11] R. Sine, Remarks on the example of Alspach, in: Nonlinear Analysis and Applications, Dekker, New York, 1982, 237-241.

Department of Mathematics and Statistics

Miami University

Oxford, OH 45056, U.S.A.

E-mail: dowlinpn@muohio.edu

Department of Mathematics and Statistics

Oakland University

Rochester, MI 48309, U.S.A.

E-mail: turett@oakland.edu
Department of Mathematics University of Pittsburgh Pittsburgh, PA 15260, U.S.A.

E-mail: lennard+@pitt.edu

Received March 28, 2006

Revised version February 15, 2007 